\title{
MODELAGEM, SIMULAÇÃO E ANÁLISE DO SISTEMA DE EVAPORADORES DE MISCELA DE UMA DESTILARIA DE OLEO DE SOJA UTILIZANDO O SOFTWARE HYSYS
}

\author{
E. R. A. PRADO ${ }^{1}$, A. TROMBETA ${ }^{1}$, L. V. DURANTE ${ }^{1}$, P. R. PARAISO ${ }^{1}$ e L. M. M. \\ $\mathrm{JORGE}^{1}$ \\ ${ }^{1}$ Universidade Estadual de Maringá, Departamento de Engenharia Química \\ E-mail para contato: dudarasi@yahoo.com.br
}

\begin{abstract}
RESUMO - O processo de obtenção de óleo de soja é o mais importante setor da agroindústria brasileiro. A redução de custos é fundamental para a competitividade no mercado global, essa redução pode ocorrer na etapa de destilação, onde ocorre um grande consumo de energia, sendo esta constituída por três evaporadores e um "stripper" com objetivo de separar o óleo bruto do solvente hexano. A simulação e analise, utilizando o HYSYS, das entradas e saídas das correntes dos evaporadores, se torna atrativa devido à possibilidade de analisar estratégias para redução de consumo de vapor. Neste contexto, a partir de um modelo de evaporadores validados com os dados reais do processo, analisouse a simulação de dois sistema, concorrente e contra corrente, dos evaporadores de miscela. Os resultados obtidos pelo HYSYS revelam que quando a miscela e o gás estão em contra corrente há uma menor consumo de vapor, sem alterar a eficiencia do processo de obtenção de oleo de soja.
\end{abstract}

\section{INTRODUÇÃO}

A industrialização de oleaginosas constitui-se num dos importantes setores agroindustrial. No abastecimento e distribuição mundial das oleaginosas, a soja se destaca como a principal, onde em 2011, correspondeu a 58,09\% de todas as oleaginosas produzidas mundialmente. No abastecimento e distribuição mundial da soja, o Brasil é o segundo maior produtor, correspondendo a 28,93\% da produção mundial, ficando atrás dos Estados Unidos da América, que corresponde a 31,98\%. A China em 2011, representou 60,13\% de toda importação mundialmente. O Brasil é o segundo pais, em exportação de soja, correspondendo por $32,41 \%$ de toda a exportação, ficando atrás dos Estados Unidos da América, que representam a 44,21\%. No processamento da soja, a China em 2011, correspondeu a 26,21\% de toda a soja processada no mundo (Oilseeds, 2012).

De acordo com Fernandes-Junior (2009), na indústria de processamento de óleos vegetais, nos últimos cinco anos houve um aumento de capacidade instalada de processamento de $35 \%$ no Brasil, sendo que no estado do Paraná, este crescimento foi de 21,4\%. A continuidade deste crescimento será possível com a otimização dos custos industriais, com relação ao custo da energia necessária no processo. 
A produção de óleo de soja é um processo complexo e envolve recepção, tratamento, transporte e armazenamento da matéria prima. Na extração do óleo por solvente (hexano), a mistura óleo vegetal e solvente é denominada de miscela. Na evaporação e destilação "flash", ocorrer à concentração do óleo na miscela. Para separar os dois componentes, acontecendo um alto consumo de energia (Paraiso, 2001). Abaixo esta o processo de destilação da miscela, onde obtem óleo vegetal bruto.

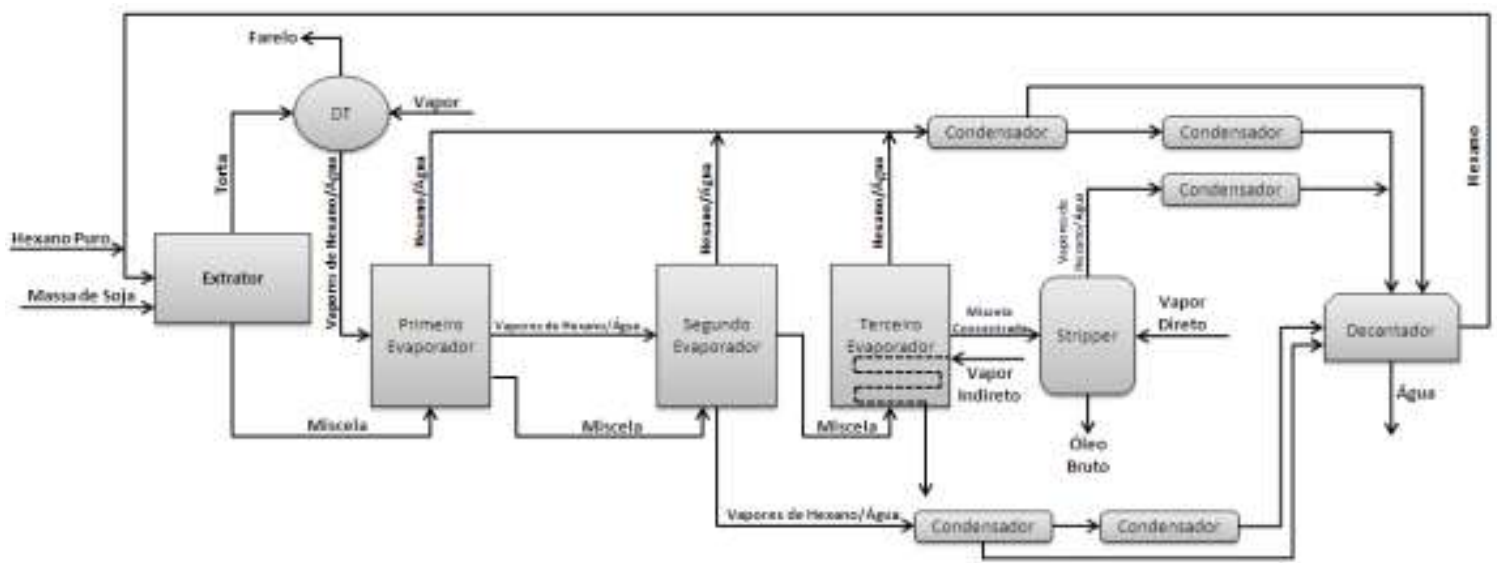

Figura 1 - Fluxograma do processo de destilação da miscela.

A destilação da miscela é composta por colunas de evaporação e stripper, demonstrado na Figura 1. Nestas operações, são utilizadas aproximadamente $40 \mathrm{Kg}$ de vapor / ton. de soja processada (Jongeneelen, 1976). Os evaporadores, exerce a função de trocadores de calor, nos quais o vapor de aquecimento flui na parte externa dos tubos, no sentido de cima para baixo, e a miscela escoa na parte interna dos tubos, no sentido de baixo para cima. A miscela entra no primeiro evaporador com concentração de $25,0 \%$, após passar por dois evaporadores, a concentração é de $65 \%$ de óleo e após o terceiro evaporador a concentração é de $97 \%$ de óleo.

A modelagem e simulação de processos utilizando simulador como o HYSYS, auxilia na determinação das melhores condições de processamento, visando à redução de custos, proteção do meio ambiente e consumidor nas indústrias, propiciando um consumo menor de energia e uma recuperação de hexano. De acordo com Jorge, et al (2010), seguindo a tendência dos simuladores comerciais, o HYSYS possui blocos de simulação voltados à petroquímica, evidenciando a comprovação da aplicabilidade dos simuladores a outros seguimentos, como o setor de óleos vegetais.

Santander, et. al (2010), emulou o comportamento dos óleos vegetais, por meio de compostos das misturas que o compõem. Foram realizadas medições experimentais das propriedades dos compostos em estudos. Nas simulações utilizou o modelo termodinâmico NRTL, os parâmetros ausentes no simulador foram estimados pelos métodos UNIFAC.

O modelo termodinâmico molecular NRTL (nonrandom twoliquids), proposto por Sandler (2003), é uma extensão da equação de Wilson e utiliza a mecânica estatística e o conceito de composição local para representar a estrutura líquida. A diferença com o modelo de Wilson é que o modelo NRTL é aplicável com miscibilidade parcial, permitindo representar os equilíbrios líquido-vapor, líquido-líquido e líquido-líquido-vapor, este modelo 
é usado em misturas líquidas não eletrolíticas, representando as propriedades de misturas não ideais melhores que outras equações, como por exemplo, Margules e Van Laar.

Este trabalho teve como objetivo principal simular e analisar a operação de evaporação da miscela de soja, utilizando o software HYSYS, comparando os resultados para processos concorrente e contracorrentes nos evaporadores.

\section{MODELAGEM DO SISTEMA DE EVAPORAÇÃO NO HYSYS}

No modelo foram utilizados módulos disponíveis no HYSYS. O evaporador foi desenvolvido, a partir da associação de um trocador de calor tipo multi tubular e um vaso separador flash. Esta ideia foi inicializada por Foust, et al (1982) referindo o evaporador, constituído por um trocador de calor e um separador de fase vapor do líquido em ebulição. Este mesmo conceito foi utilizado por Jorge (2010), analisando a concentração do caldo de cana de açúcar, utilizando simulador HYSYS.

O óleo de soja é constituído por $95 \%$ de ácidos orgânicos. As estruturas moleculares do óleo de soja, não estão disponíveis no HYSYS, utilizou a avaliação de distribuição de grupos de moléculas, do método UNIFAC. Montadas moléculas hipotéticas Oléico, Linoleico, Linolênico, Palmitoléico, Araquidico, Esteárico, Palmítico, Mirístico, Láurico e Esqualeno.

$\mathrm{Na}$ modelagem dos evaporadores, foi utilizado o óleo de soja processado na Cooperativa Agroindustrial COCAMAR. Os demais parâmetros dos ácidos graxos foram estimados pelo HYSYS. Para montagem do modelo do processo dos evaporadores de miscela, os equilíbrios de fases e coeficientes de atividades, foi estimados utilizando os modelos termodinâmicos NRTL e Peng-Robinson, cujos mesmos são selecionados no software utilizado. O conceito de NRTL baseia-se na hipótese de Wilson que a concentração local em torno de uma molécula é diferente do volume do material. O modelo NRTL é aplicável a sistemas com miscibilidade parcial, representando equilíbrios líquido-vapor, líquido-líquido e líquido-líquido-vapor.

\subsection{Modelagem do sistema de evaporação no Hysys em concorrente}

Seguindo o mesmo conceito, desenvolveu-se um modelo do sistema de evaporação, para a concentração da miscela de um processo de evaporação com alimentação e concorrente. A figura abaixo demonstra o modelo do processo de concentração de miscela, montado no HYSYS. 


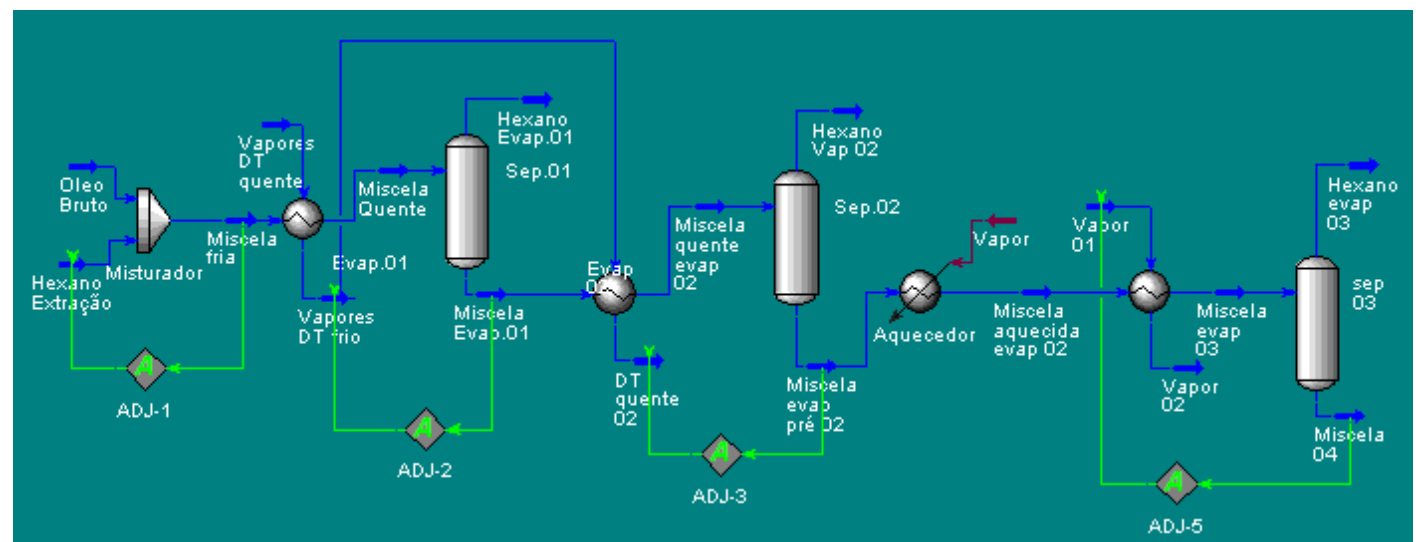

Figura 2 - Modelo do sistema em concorrente de evaporação de miscela no HYSYS.

Na simulação do modelo representado pela Figura 2, o trocador de calor representa a calandra do evaporador e de acordo com Tema (1998), admite-se um passo no casco e um passo nos tubos, com orientação vertical, espaçamento máximo entre os espelhos e classificação A-E-L, conforme as normas apresentadas.

O coeficiente global de troca térmica foi estimado pelo HYSYS, foi desenvolvido quatro rotinas de convergência na simulação da modelagem dos evaporadores, fixando as concentrações de hexano em cada corrente de saída dos separadores, ajustando a vazão mássica de hexano, na entrada do extrator, as frações de vapor na saída do gás quente do DT, nos dois evaporadores iniciais e a vazão mássica de vapor saturado na entrada do ultimo evaporador.

Os parâmetros operacionais, utilizados como bases para a simulação do modelo representado pela Figura 2 estão apresentadas na tabela 1 .

Tabela 01 - Parâmetros operacionais da COCAMAR, utilizados nas simulações

\begin{tabular}{|c|c|c|c|}
\hline Descrição & $1^{\mathbf{o}}$ & $2^{\mathbf{o}}$ & $3^{\mathbf{o}}$ \\
\hline Vazão Mássica da Miscela na entrada $(\mathrm{Kg} / \mathrm{h})$ & 87.190 & - & - \\
\hline Fração mássica do óleo na entrada $(\%)$ & 23,5 & - & - \\
\hline Vazão mássica do Hexano na saída $(\mathrm{Kg} / \mathrm{h})$ & 53.040 & - & - \\
\hline Temperatura da Miscela, na entrada $\left({ }^{\circ} \mathrm{C}\right)$ & 58 & 60 & 110 \\
\hline Temperatura corrente de aquecimento $\left({ }^{\circ} \mathrm{C}\right)$ & 80 & 75 & 180 \\
\hline Pressão de Operação $\left(\mathrm{mmHg}^{2}\right)$ & 520 & 520 & 520 \\
\hline Área de troca térmica $\left(\mathrm{m}^{2}\right)$ & 1066 & 350 & 266 \\
\hline Número de Tubos & 677 & 915 & 556 \\
\hline Comprimento dos tubos $(\mathrm{m})$ & 10 & 5 & 6 \\
\hline
\end{tabular}

\subsection{Modelagem do sistema de evaporação no Hysys em contracorrente}

Seguindo o mesmo conceito, desenvolveu-se um modelo do sistema de evaporação para a concentração da miscla de um processo de evaporação com alimentação em contracorrente. A figura abaixo monstra o modelo de processo de concentração de miscela, no HYSYS. 


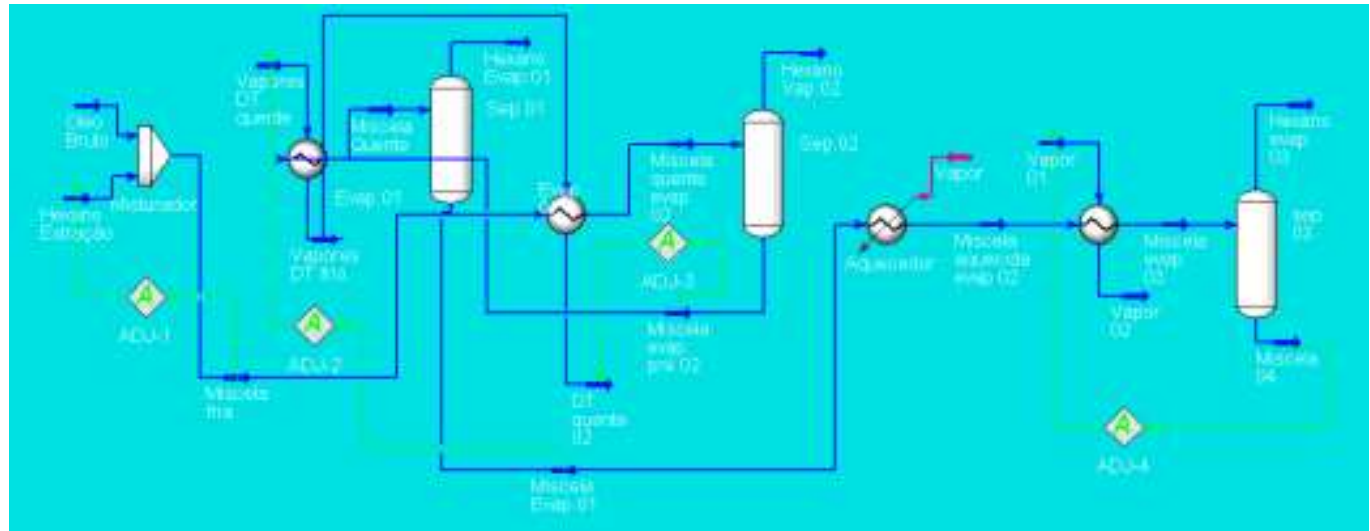

Figura 3 - Modelo do sistema em contracorrente de evaporação de miscela no HYSYS.

Na simulação do modelo representado pela Figura 3, admite-se um passo no casco e um passo nos tubos, com orientação vertical, espaçamento máximo entre os espelhos e classificação A-E-L. Assim como no modelo anterior o coeficiente global de troca térmica foi deixado como parâmetro estimado pelo HYSYS, foi necessário desenvolver quatro rotinas de convergência na simulação da modelagem dos evaporadores, fixando as concentrações de hexano em cada corrente de saída dos separadores, ajustando a vazão mássica de hexano, na entrada do extrator, as frações de vapor na saída do gás quente do DT, nos dois evaporadores iniciais e a vazão mássica de vapor na entrada do ultimo evaporador.

Os parâmetros operacionais da COCAMAR nessa nova situação, utilizados para a simulação do modelo representado pela Figura 3 estão apresentadas na tabela 2.

Tabela 02 - Parâmetros operacionais da COCAMAR, utilizados nas simulações

\begin{tabular}{|c|c|c|c|}
\hline Descrição & $1^{\circ}$ Evaporador & $2^{\circ}$ Evaporador & $3^{\circ}$ Evaporador \\
\hline Vazão Mássica de Miscela na entrada $(\mathrm{Kg} / \mathrm{h})$ & 77.700 & 47860 & 32.510 \\
\hline Fração mássica do óleo na entrada $(\%)$ & 25,74 & 41,8 & 61,56 \\
\hline Vazão mássica de Hexano na saída $(\mathrm{Kg} / \mathrm{h})$ & 29.840 & 15.350 & 12.100 \\
\hline Temperatura da Miscela na entrada $\left({ }^{\circ} \mathrm{C}\right)$ & 71,04 & 59,37 & 121,5 \\
\hline Pressão de Operação $(\mathrm{mmHg})$ & 520 & 520 & 520 \\
\hline Área de troca térmica $(\mathrm{m} 3)$ & 1066 & 350 & 266 \\
\hline Número de Tubos & 677 & 915 & 556 \\
\hline Comprimento dos tubos $(\mathrm{m})$ & 10 & 5 & 6 \\
\hline
\end{tabular}

\section{RESULTADOS}

\subsection{Modelo concorrente}

Os resultados obtidos na simulação, utilizando as condições operacionais da Tabela 1, foram comparados com os dados do processo industrial da COCAMAR, demonstrados nas tabelas 03 e 04 , para o $1^{\circ}, 2^{\circ}$ e $3^{\circ}$ evaporador, respectivamente. Considerando constante a vazão de entrada da miscela, sendo utilizada a mesma vazão na simulação no HYSYS, na entrada de miscela do segundo e terceiro evaporador houve um desvio de $0 \%$ e $0,03 \%$, respectivamente. Para o hexano na saída do segundo e terceiro evaporadores, o desvio observado foi de $0,49 \%$ e $0,05 \%$, indicando proximidade dos valores reais do processo. 
Tabela 03 - Avaliação das previsões do HYSYS, medidas operacionais do $1^{\circ}$ Evaporador

\begin{tabular}{|c|c|c|c|}
\hline \multirow{2}{*}{ Variáveis } & \multicolumn{3}{|c|}{$1^{\circ}$ Evaporador } \\
\cline { 2 - 4 } & COCAMAR & HYSYS & Desvio \\
\hline Vazão mássica da miscela na saída $(\mathrm{Kg} / \mathrm{h})$ & 34.150 & 34.150 & $0 \%$ \\
\hline Fração mássica de óleo na saída $(\%)$ & 60 & 60 & $0 \%$ \\
\hline Temperatura da miscela na saída $\left({ }^{\circ} \mathrm{C}\right)$ & 61 & 61,99 & $1,62 \%$ \\
\hline Vazão do fluido de aquecimento $(\mathrm{Kg} / \mathrm{h})$ & - & 34.870 & - \\
\hline Coeficiente de transferência de calor $\left(\mathrm{Kcal} / \mathrm{h} \cdot \mathrm{m}^{2}{ }^{2}{ }^{\circ} \mathrm{C}\right)$ & - & 282,99 & - \\
\hline
\end{tabular}

Tabela 04 - Avaliação das previsões do HYSYS, medidas operacionais do $2^{\circ}$ e $3^{\circ}$ Evaporador

\begin{tabular}{|c|c|c|c|c|c|c|}
\hline \multirow{2}{*}{ Variáveis } & \multicolumn{3}{|c|}{$3^{\circ}$ Evaporador } & \multicolumn{3}{c|}{$2^{\circ}$ Evaporador } \\
\cline { 2 - 7 } & Cocamar & Hysy & Desvio & Cocamar & Hysy & Desvio \\
\hline Vazão mássica miscela entra (ton/h) & 31,51 & 31,5 & $0,03 \%$ & 34,15 & 34,15 & $0 \%$ \\
\hline Vazão mássica miscela sai (ton/h) & 21,00 & 21,0 & $0,05 \%$ & 31,51 & 31,52 & $0,03 \%$ \\
\hline Vazão mássica Hexano sai (ton/h) & 10,50 & 10,5 & $0,05 \%$ & 2,64 & 2,63 & $0,49 \%$ \\
\hline Fração mássica óleo entra (\%) & 65 & 65 & $0 \%$ & 60 & 60 & $0 \%$ \\
\hline Fração mássica óleo sai (\%) & 97,5 & 97,5 & $0 \%$ & 65 & 65 & $0 \%$ \\
\hline Temperatura miscela sai ( $\left.{ }^{\circ} \mathrm{C}\right)$ & 110 & 111 & $0,91 \%$ & 59 & 58,43 & $0,97 \%$ \\
\hline Vazão fluido aquecimento (ton/h) & - & 2,78 & - & - & 34,87 & - \\
\hline $\begin{array}{c}\text { Coeficiente de transferência de } \\
\left.\text { calor (Kcal/h.m }{ }^{2}{ }^{\circ} \mathrm{C}\right)\end{array}$ & - & 71,32 & - & - & 35,60 & - \\
\hline
\end{tabular}

Comparando a fração mássica de óleo, observa-se que não houve desvio na concentração do óleo na miscela de saída dos três evaporadores, como podemos observar nas tabelas 3 e 4 . Comparando as temperaturas na saída dos evaporadores, observa-se um desvio de $-1,62 \%, 0,97 \%$ e $0,91 \%$ no primeiro, segundo e terceiro evaporador, respectivamente. Comparando as vazões de miscela e hexano e a concentração de óleo nas correntes de saída, houve um desvio pequeno, mesmo sendo todos os parâmetros dos componentes hipotéticos do óleo de soja aproximados a partir das frações dos ácidos graxos estimados pelo HYSYS.

\subsection{Modelo Contracorrente}

Os resultados obtidos na simulação, utilizando as condições operacionais da Tabela 1, foram comparados com os dados do processo industrial da COCAMAR, cujos resultados e desvios percentuais em relação as medidas no processo, encontram-se nas tabelas 06 e 07, para o $1^{\circ}, 2^{\circ}$ e $3^{\circ}$ evaporador, respectivamente, assim como no modelo em concorrente.

Tabela 06 - Avaliação das previsões do HYSYS, medidas operacionais do $1^{\circ}$ Evaporador

\begin{tabular}{|c|c|c|c|}
\hline \multirow{2}{*}{ Variáveis } & \multicolumn{3}{|c|}{$1^{\circ}$ Evaporador } \\
\cline { 2 - 4 } & Cocamar & Hysys & Desvio \\
\hline Vazão mássica da miscela na saída (ton/h) & 47,86 & 47,86 & $0 \%$ \\
\hline Fração mássica de óleo na saída $(\%)$ & 41,8 & 41,8 & $0 \%$ \\
\hline Temperatura da miscela na saída $\left({ }^{\circ} \mathrm{C}\right)$ & 70 & 71,04 & $1,46 \%$ \\
\hline Vazão do fluido de aquecimento $($ ton $/ \mathrm{h})$ & - & 26,98 & - \\
\hline Coeficiente de transferência de calor $\left(\mathrm{Kcal} / \mathrm{h} \cdot \mathrm{m}^{2}{ }^{\circ} \mathrm{C}\right)$ & - & $1.048,48$ & - \\
\hline
\end{tabular}


Tabela 07 - Avaliação das previsões do HYSYS, medidas do $2^{\circ}$ e $3^{\circ}$ Evaporadores

\begin{tabular}{|c|c|c|c|c|c|c|}
\hline \multirow{2}{*}{ Variáveis } & \multicolumn{3}{|c|}{$3^{\circ}$ Evaporador } & \multicolumn{3}{c|}{$2^{\mathbf{o}}$ Evaporador } \\
\cline { 2 - 7 } & Cocamar & Hysys & Desvio & Cocamar & Hysys & Desvio \\
\hline Vazão miscela entra (ton/h) & 32,00 & 32,51 & $1,57 \%$ & 47,86 & 47,86 & $0 \%$ \\
\hline Vazão miscela sai (ton/h) & 20,50 & 20,41 & $0,44 \%$ & 31,51 & 32,51 & $0,03 \%$ \\
\hline Vazão Hexano na sai (ton/h) & 11,50 & 12,10 & $4,95 \%$ & 15,10 & 15,35 & $1,63 \%$ \\
\hline Fração mássica óleo entra (\%) & 61,53 & 61,53 & $0 \%$ & 41,8 & 41,8 & $0 \%$ \\
\hline Fração mássica óleo sai (\%) & 98 & 98 & $0 \%$ & 61,53 & 61,53 & $0 \%$ \\
\hline Temperatura miscela sai ( $\left.{ }^{\circ} \mathrm{C}\right)$ & 118 & 119,8 & $1,50 \%$ & 57 & 56,92 & $0,14 \%$ \\
\hline Vazão fluido (ton/h) & - & 5,88 & - & - & 26,98 & - \\
\hline $\begin{array}{l}\text { Coeficiente transferência de } \\
\left.\text { calor(Kcal/h.m }{ }^{2} .{ }^{\circ} \mathrm{C}\right)\end{array}$ & - & 369,2 & - & - & 96,04 & - \\
\hline
\end{tabular}

Houve um desvio pequeno nas variáveis, mesmo os componentes hipotéticos do óleo de soja aproximados a partir dos ácidos graxos estimados no HYSYS, os resultados foram bons.

\section{Análise dos modelos em relação ao consumo de vapor}

A comparação destes dois modelos apresentados deve ser analisada de forma econômica, simulamos para encontrar a vazão de vapor na entrada dos evaporadores. A partir das simulações foi quantificado o vapor gasto em cada modelo e o custo que isso representa.

Tabela 09 - Análise gasto de vapor dos modelos concorrente e contracorrente

\begin{tabular}{|c|c|c|c|c|}
\hline \multirow{2}{*}{ Simulações } & \multicolumn{2}{|c|}{ Concorrente } & \multicolumn{2}{c|}{ Contracorrente } \\
\cline { 2 - 5 } & Gases DT $(\mathrm{kg} / \mathrm{h})$ & Vapor $(\mathrm{kg} / \mathrm{h})$ & Gases DT $(\mathrm{kg} / \mathrm{h})$ & Vapor $(\mathrm{kg} / \mathrm{h})$ \\
\hline 1 & $64.550,00$ & $2.472,00$ & $33.650,00$ & $2.914,00$ \\
\hline 2 & $69.210,00$ & $2.389,00$ & $28.850,00$ & $2.989,00$ \\
\hline 3 & $69.380,00$ & $2.723,00$ & $29.940,00$ & $3.403,00$ \\
\hline 4 & $70.080,00$ & $2.391,00$ & $28.930,00$ & $3.252,00$ \\
\hline 5 & $61.940,00$ & $2.430,00$ & $29.130,00$ & $3.355,00$ \\
\hline 6 & $63.140,00$ & $2.438,00$ & $31.240,00$ & $2.760,00$ \\
\hline 7 & $69.740,00$ & $2.318,00$ & $31.070,00$ & $4.155,00$ \\
\hline 8 & $62.820,00$ & $2.594,00$ & $26.600,00$ & $2.805,00$ \\
\hline 9 & $67.580,00$ & $2.271,00$ & $25.940,00$ & $3.015,00$ \\
\hline 10 & $67.230,00$ & $2.549,00$ & $27.880,00$ & $3.188,00$ \\
\hline $\begin{array}{c}\text { Vapordia } \\
\text { sem hexágua }\end{array}$ & $66.580,00$ & $2.431,92$ & $29.171,54$ & $3.158,08$ \\
\hline O mexano & $7.536,12$ & $2.431,92$ & $3.301,90$ & $3.158,08$ \\
\hline
\end{tabular}

O modelo em concorrente gasta 56,19\% mais vapor do dessolventisador tostador que em contracorrente. O segundo modelo gasta $29,86 \%$ mais vapor do que em concorrente para o $3^{\circ}$ evaporador. Tendo a referencia o custo do vapor industrial de 2012, em R \$ 62 / tonelada.

Tabela 11 - Comparação custo entre os modelos

\begin{tabular}{|c|c|c|}
\hline Modelo & Concorrente & Contracorrente \\
\hline Vazão de vapor $(\mathrm{kg} / \mathrm{h})$ & 9968,04 & 6459,98 \\
\hline Custo econômico $(\mathrm{R} \$ \mathrm{~h})$ & 618,02 & 400,52 \\
\hline Custo econômico anual $(\mathrm{R} \$ / \mathrm{ano})$ & $5.413 .843,85$ & $3.508 .542,85$ \\
\hline
\end{tabular}


A diferença mostra que o modelo em contracorrente economiza $\mathrm{R} \$ 1.905 .301,00$.

\section{CONCLUSÕES}

A utilização de um sistema de simulação permitiu a analise de gasto de vapor dos modelos. O modelo em contracorrente gera economia, sem perder a qualidade do produto. A modelagem e simulação do processo de evaporação da miscela, utilizando o HYSYS, conduziram a valores coerentes com as condições reais do processo industrial, logo pode se utilizar como ferramenta computacional no processo de analise, simulação e otimização da operação de destilação do óleo de soja, para a concentração da miscela nos três evaporadores.

Após a avaliação dos dois tipos de modelo, conclui-se que houve uma redução do gasto de vapor d'água em 35,19\%, gerando uma economia de vapor d'água de aproximadamente R \$1.905.301,00 no ano. A partir da simulação e analise dos resultados, foi realizado a implementação do fluxo contracorrente, na etapa da concentração de miscela, na extração de óleo vegetal.

\section{REFERÊNCIAS}

FERNANDES-JUNIOR, C. C., Integração energética da etapa de extração de óleo de soja, utilizando a análise Pinch. Unioeste, Toledo-PR (dissertação de mestrado), 2009.

FOUST, A. S.; WENZEL, L. A.; CLUMP, C. W.; ANDERSON, L. B. Princípios das Operações Unitárias, Guanabara Dois, Rio de Janeiro-RJ, p.436, 1982.

JONGENEELEN, H. P. J., Energy Conservation in Solvent Extraction Plants, JAOCS Journal of the American Oil Chemists Society, vol.53, p. 291-308, 1976.

JORGE, L. M. M.; RIGHETTO, A. R.; POLLI, P. A.; SANTOS, O. A. A.; MACIEL FILHO, R., Simulation and analysis of a sugarcane juice evaporation system. Journal of Food Engineering, 351-359, 2010.

OILSEEDS - World Markets and Trade. Washington: USDA, Jan. 2012.

PARAÍSO, P. R., Modelagem e Análise do Processo de Obtenção do Óleo de Soja, Unicamp, Campinas-SP (Tese de Doutorado), 2001.

SANDLER, S. I., Chemical and Engineering Thermodynamics, $2^{\text {nd }}$ ed., John Wiley and Sons, 2003.

SANTANDER, C.M.G.; COSTA, ALINE C.; MACIEL FILHO, R. Pretreatment of sugarcane bagasse with phosphoric and sulfuric diluted acid for fermentable sugar production by enzymatic hydrolysis. Chemical Engineering Transactions, v. 20, p. 321-326, 2010.

TEMA, Standards of the tubular exchange manufactures association, Enchanger Manufactures, 7. Ed., Nova York, 1998. 